(c) 2020 INIE

Universidad de Oviedo

Dirección postal:

Edif. de Servicios Múltiples, $1^{\text {a }}$ planta Campus de Humanidades, C/ Amparo Pedregal, s/n, 33011 Oviedo.

Administración: inie@uniovi.es

Tel.: 985105008

\section{Contacto principal:} Instituto de Investigación e Innovación Educativa inie@uniovi.es

Secretaría de la Revista:

Edif. de Servicios Múltiples, $1^{\text {a }}$ planta Campus de Humanidades, C/ Amparo Pedregal, s/n, 33011 Oviedo.

e-mail:inie@uniovi.es

Contactos de asistencia: María Mercedes García Cuesta e-mail: mgcuesta@uniovi.es Verónica Cañal Fernández e-mail: secinie@uniovi.es

\section{Publicado por:} Ediuno

Ediciones de la Universidad de Oviedo Servicio de Publicaciones de la Universidad de Oviedo

Campus de Humanidades. Edificio de Servicios.

33011 Oviedo (Asturias) Tel. 985109503 Fax 985109507 http: www.uniovi.es/publicaciones servipub@uniovi.es

\section{Edición Digital:} REUNIDO

www.unioviedo.es/reunido

Todos los derechos reservados. De conformidad con lo dispuesto en la legislación vigente, podrán ser castigados con penas de multa y privación de libertad quienes reproduzcan o plagien, en todo o en parte, una obra literaria, artística o científica, fijada en cualquier tipo de soporte, sin la preceptiva autorización.
Director

Günter L. Huber, Profesor emérito de la Universidad de Tübingen, Waden - Witenberg, Alemania

Codirector

Emilio Álvarez-Arregui, Universidad de Oviedo, España

\section{Editora Asociada}

Verónica Cañal Fernández, Universidad de Oviedo, España

\section{Editores Temáticos}

José Pablo Paredes Sánchez, Universidad de Oviedo, España Alejandro Rodríguez-Martín, Universidad de Oviedo, España Beatriz Rodríguez Ruiz, Universidad de Oviedo, España

\section{Gestión y Administración} María Mercedes García Cuesta, Universidad de Oviedo, España

\section{Consejo Técnico}

Alberto Fernández Costales, Universidad de Oviedo, España

Lucía Álvarez Blanco, Universidad de Oviedo, España

Beatriz A. Grossi Sampedro, Universidad de Oviedo, España Aránzazu Valdés González, Universidad de Oviedo, España Ana Rosa Arias Gago, Universidad de León, España

Francisca González Gil, Universidad de Salamanca, España

Eva María Iniesta Mena, Universidad de Oviedo, España

Jesús Alemán Falcón, Universidad de las Palmas de Gran Canaria, España

José Luis Belver Domínguez, Universidad de Oviedo, España

José Manuel Montejo Bernardo, Universidad de Oviedo, España

María Mitre Aranda, Universidad de Oviedo, España

María Paulina Viñuela Hernández, Universidad de Oviedo, España

Marta Soledad García Rodríguez, Universidad de Oviedo, España

Mónica Herrero Vázquez, Universidad de Oviedo, España

Susana Agudo Prado, Universidad de Oviedo, España

\section{Revista Científica Internacional Indexada}

(Indexed International Scientific Journal)

\section{Directorios de revistas científicas:}

DICE (Difusión y Calidad Editorial de Revistas)

Dulcinea

Elektronische Zeitschriftenbibliothek (Universität Regensburg)

IRESIE (Índice de Revistas de Educación Superior e Investigación de México)

ISOC (Centro de Información y Documentación Científica del Consejo Superior de Investigaciones Científicas)

REDINED (Red de Información Educativa)

Ulrich's International Periodicals Directory

Zeitschriftendatenbank (Biblioteca Nacional de Berlin)

ScienceDirect

SCOPUS

WOS - ESCI

Plataformas de evaluación de revistas:

ANEP (Agencia Nacional de Evaluación y Prospectiva)

CARHUS

CIRC (Clasificación Integrada de Revistas Científicas)

Google Scholar Metrics

IN-RECS (Índice de Impacto de Revistas Españolas de Ciencias Sociales)

MIAR (Matriu d'informació per a l'Avaluació de Revistes)

Latindex. Catálogo Selectivo

RESH (Revistas Españolas de Ciencias Sociales y Humanidades)

\section{Buscadores de literatura científica en Open Access:}

Dialnet.

$D O A J$, Scientific Commons

Google Scholar (GSM)

OAISTER

The Library of Congress

Scirus 


\section{Comité Científico Internacional}

Alessandro Pepe. Universidad de Milán-Bicocca. Italia

Álvaro Marchesi Ullastres. Universidad Complutense de Madrid, España

Alpesh Maisuria. University of East London - London, United Kingdom

Anais Janyan. Claremont Graduate University - California, EE.UU.

Andrew Richards. University of Exeter, United Kingdom

Andreas Demetriou. University of Nicosia, Cyprus

Catherine Blaya. Université Nice Sophia Antipolis, Francia

Catherine Snow. Harvard University, EE.UU.

Charlina Gozali. Claremont Graduate University - California, EE.UU.

Cristina Stringher. National Italian Institute for Educational Evaluation of Instruction and Training (INVALSI), Roma - Italia Cristine W. Kang. Claremont Graduate University - California, EE.UU.

David Scanlon. Boston College, EE.UU.

Donatella Persico. Instituto Tecnologie Didattiche - Consiglio Nazionale Ricerche, Italia

E. Wayne Ross. University of British, Columbia Vancouver - Canada.

Fabio Leoni. University of Locarno, Suiza

Frida Díaz Barriga. Universidad Nacional Autónoma de México. México

Faye Antoniou. University of Thessaly, Grecia

Fernando Ribeiro Gonçalves. Universidade do Algarve, Portugal Francesca Caena. Università Ca 'Foscari Venezia, Italia

Francesco Tonucci. Laboratory of Psychology of Child Participation, Italia

Gert Rijlaarsdam. University of Ámsterdam, Holanda

Gina Conti-Ramsden. University of Manchester, Reino Unido

Herberg J. Walberg. University of Illinois, EE.UU.

Ignacio Aguaded. Universidad de Huelva, España

Ioannis Agaliotis. University of Macedonia, Grecia

Jairo Lugo. Universidad de Leeds, Reino Unido

Jarkko Hautamäki. University of Helsinki. Helsinki.

Jean-Francois Hugues. Innobridge, Switzerland

Joyce L. Epstein. Johns Hopkins University, Baltimore, EE.UU.

Julio Cabero Almenara. Universidad de Sevilla, España

Keneth Johnson. University of Belfast, Reino Unido

Leandro S. Almeida. Universidade do Minho, Portugal

Loizos Symeou. Associate Professor, European University Cyprus, Cyprus

Loredana Addimando. University of Applied Sciences and Arts of Southern Switzerland, Switzerland

Massimo Marcuccio. Universidad de Bolonia. Italia

María Flores Tena. UNAM, México

María Savva. European University Cyprus, Cyprus

María Soledad Ramírez Montoya. Tecnológico de Monterrey, México

Matthias Grunke. University of Cologne, Alemania

Michelle Gautreaux. University of British, Columbia Vancouver - Canada

Nathalis Wamba. University of New York, EE.UU.

Nina Hidalgo. Universidad Autónoma de México, México

Pedro José Arrifano Tadeu. Instituto Politécnico de Guarda, Portugal

Raquel Amaya Martínez González. Universidad de Oviedo, España

Ricardo Valenzuela González. Tecnológico de Monterrey, México

Rollande Deslandes. Université du Québec à Troi-Rivières, Canadá

Roger Azevedo. McGill University, Canadá

Rosemary Hipkins. New Zealand Council for Educational Research. New Zealand

Rosemary Tannock. University of Toronto, Canadá

Rusell Barkley. University of Masachussets, EE.UU.

Ruth Deakin Crik. University of Bristol, United Kingdom

Sirkku Kupiainen. University of Helsinki. Helsinki

Steven B. Sheldon. Johns Hopkins University - Baltimore, EE.UU.

Shirlee Mae Mamaril Choe. Claremont Graduate University - California, EE.UU.

Susan J. Paik, Claremont Graduate University - California, EE.UU.

Tony Booth. University of Cambridge, United Kingdom

Valéry Chanal. University of Grenoble Alpes, CNRS. Francia

Vito Carioca. Instituto Politécnico de Beja, Portugal

Xavier Arreguit. General Director of the Innovation Acceleration Platform ,"Innobridge" Switzerland

Zafra Lerman. Columbia College Chicago, EE.UU. 
Alejandro Escudero Santana. Universidad de Sevilla, España Alex Ibáñez Etxeberría. Universidad del País Vasco, España Alfonso Barca Lozano. Universidad de A Coruña, España Amparo Moreno. Universidad Autónoma de Madrid, España Ana Belén Bernardo Gutiérrez. Universidad de Oviedo, España Ana Castro Zubizarreta. Universidad de Cantabria, España Ana María Castaño. Universidad de Oviedo, España Ana María Duarte Hueros. Universidad de Huelva, España Ana Pérez Escoda. Universidad Internacional de la Rioja, España Anabel Moriña. Universidad de Sevilla, España Ángel de Juanas. Universidad Nacional de Educación a Distancia, España Ángel García del Dujo. Universidad de Salamanca, España Ángela Barrios Fernández. Universidad Autónoma de Madrid, España Antoni J. Colom Cañellas. Universidad de las Islas Baleares, España Antonio García Correa. Universidad de Murcia, España Antonio León García Izquierdo. Universidad de Oviedo, España Antonio-Manuel Rodríguez-García Sánchez. Universidad de Granada, España Aquilina Fueyo Gutiérrez. Universidad de Oviedo, España Arantzazu Rodríguez-Fernández. Universidad del País Vasco, España Arturo Fuentes Cabrera Sánchez. Universidad de Granada, España Beatriz Triana Pérez. Universidad de La Laguna, España Blanca Miguelez-Juan. Universidad Complutense de Madrid, España Carmen Alba Pastor. Universidad de Castilla la Mancha, España Carmen Baldallo. Universidad de Huelva, España Carmen María Fernández García. Universidad de Oviedo, España Carmen Rodríguez Menéndez. Universidad de Oviedo, España Carmen Viejo Almanzón. Universidad de Córdoba, España Celestino Rodríguez Pérez. Universidad de Oviedo, España Celia Simón Rueda. Universidad Autónoma de Madrid, España Clara Romero Pérez. Universidad de Sevilla, España Concepción Domínguez Garrido. UNED, España Covadonga Rodríguez Cimadevilla. Universidad de Oviedo, España Cristina Mercader Juan. Universidad Autónoma de Barcelona, España David Álvarez García. Universidad de Oviedo, España

Dolors Forteza Forteza. Universitat de les Illes Balears, España

Edgar Iglesias. Universidad de Girona. España

Eduardo García Jiménez. Universidad de Sevilla, España Eduardo Vicente Dopico Rodríguez. Universidad de Oviedo, España Elena Martín Pastor. Universidad de Salamanca, España EliasVived Conte. Universidad de Zaragoza, España Enrique Javier Díez Gutiérrez. Universidad de León, España Ernesto López Gómez. Universidad Nacional de Educación a Distancia (UNED), España Esperanza Ceballos Vacas. Universidad de La Laguna, España Eva María Iniesta Mena. Universidad de Oviedo, España Eva Vicente Sánchez. Universidad de Zaragoza, España Fran J. García-García, Universidad de Valencia, España Francisco Javier García-Castilla. Universidad Nacional de Educación a Distancia. España. Francisco Javier Murillo. Universidad Autónoma de Madrid, España

Francisco Javier Rodríguez Díaz. Universidad de Oviedo, España

Fuensanta Cerezo Ramírez. Universidad de Murcia, España Gerardo Echeíta Sarrionandia. Universidad de Autónoma de Madrid, España Gonzalo Musitu Ochoa. Universidad Pablo Olavide. España Inés López Manrique. Universidad de Oviedo, España Inmaculada Berlanga. Universidad de la Rioja, España Inmaculada Orozco. Universidad de Sevilla, España Irina Salcines Talledo. Universidad de Cantabria, España Isabel Hevia Artime. Universidad de Oviedo, España Isabel María Bernedo Muñoz. Universidad de Málaga, España Isabel Morales. Universidad de Sevilla, España 
Isabel Ruíz de la Peña. Universidad de Oviedo, España Ivan De Jesús Moronta Tremols. Universidad del País Vasco, España Iván Sánchez-Cortés. Universidad Nacional de Educación a Distancia, Madrid, España Jaime Padilla. Universidad Politécnica Salesiana, Ecuador Javier González-Patiño. Universidad de Girona. España. Javier Martín Antón. Escuela Universitaria de Diseño, Innovación y Tecnología (ESNE), España Javier Urra Portillo. Centro Universitario Cardenal Cisneros, adscrito a la Universidad Complutense de Madrid. España Jesús García Albá. Universidad de Oviedo, España Jesús López Belmonte. Universidad Internacional de Valencia, España Jesús Nicasio García Sánchez. Universidad de León, España Joan Jordi Muntaner Guasp. Universitat de les Illes Balears, España Joaquín Gairín Sallán. Universidad Autónoma de Barcelona, España Joaquín Parra Martínez. Universidad de Murcia, España Jordi Adell Segura. Universitat Jaume I de Castellón, España Jorge Andrés Cortés Montalvo. Universidad de Chihuahua, México José Antonio Álvarez Castrillón. Universidad de Oviedo, España José Antonio Bueno Álvarez. Universidad Complutense de Madrid, España José Antonio Cecchini Estrada. Universidad de Oviedo, España José Antonio Torres González. Universidad de Jaén, España José Augusto Pacheco. Universidad do Minho, Portugal José Carlos Núñez Pérez. Universidad de Oviedo, España José Francisco Martín Alonzo. Universidad Pontifica Comillas de Madrid, España José Joaquín Arrieta Gallastegui. Universidad de Oviedo, España José Luis Ortega Martín. Universidad de Granada, España José Luis San Fabián Maroto. Universidad de Oviedo, España José Manuel Osoro. Universidad de Cantabria, España José Manuel Sánchez Serrano. Universidad Complutense de Madrid, España José Manuel Suárez Riveiro. Universidad Nacional de Educación a Distancia, Madrid, España José María Fernández Batanero. Universidad de Sevilla, España José María Román Sánchez. Universidad de Valladolid, España Juan Fernández Sánchez. Universidad Complutense de Madrid, España Juan Luis Fuentes. Universidad Nacional de Educación a Distancia (UNED), España Julián Pascual Díez. Universidad de Oviedo, España leva Margevica. Latvijas Universitate, Letonia Loreto Díaz Suárez. Universidad de Oviedo, España Lourdes Villalustre Martínez. Universidad de Oviedo, España Luis José Rodríguez Muñiz. Universidad de Oviedo, España Luis Mañas-Vinegra. Universidad del País Vasco, España Luis Miguel Pedrero Esteban. Universidad Pontificia de Salamanca, España Luis Miguel Romero Rodríguez. Universidad Internacional de la Rioja, España Luis Miguel Villar Angulo. Universidad de Sevilla, España Luis Torrego Egido. Universidad de Valladolid, España Luz Florinda Pérez Sánchez. Universidad Complutense de Madrid, España Manuel Deaño Deaño. Universidad de Vigo, España Manuel López Torrijo. Universidad de Valencia, España Manuel Soriano Ferrer. Universidad de Valencia, España María Amor Pérez Rodríguez. Universidad de Huelva, España María Ángeles Bravo Álvarez. Universidad de Cantabria. España María Ángeles Gomariz Vicente. Universidad de Murcia, España María Carmen González Torres. Universidad de Navarra, España María del Carmen Caldeiro Pedreira. Universidad de Cantabria, España María del Carmen Fernández Rubio. Universidad de Oviedo, España María del Carmen Huerta Guerra. National Italian Institute for Educational Evaluation of Instruction and Training (INVALSI), Roma - Italia María del Carmen Pegalajar Palomino. Universidad de Huelva, España María del Carmen Pérez Fuentes. Universidad de Almería, España María Dolores Díaz Noguera. Universidad de Sevilla, España María Esther del Moral Pérez. Universidad de Oviedo, España María Luz Cacheiro González. Universidad Nacional de Educación a Distancia, UNED, España 
María Rodríguez Palero. Universidad de Sevilla, España María Verdeja Muñiz. Universidad de Oviedo. España María Verónica Leiva Guerrero. Pontificia Universidad Católica de Chile, Chile Marisa Pereira González. Universidad de Oviedo, España Marta García Lastra. Universidad de Cantabria, España Mayka García García. Universidad de Cádiz, España Mercedes Gómez López. Universidad de Córdoba, España Mercedes González Sanmamed. Universidad de A Coruña, España Miguel Ángel Carbonero Martín. Universidad de Valladolid, España Miguel Ángel Ortiz. Universidad de Castilla la Mancha, España Miguel Calleja Puerta. Universidad de Oviedo, España Miguel Melendro. Universidad Nacional de Educación a Distancia. España Miriam Sonlleva Velasco. Universidad de Valladolid, España Moisés Esteban-Guitart. Universidad de Girona. España Mónica Alonso Alonso. Universidad de Oviedo, España Nazario Yuste Rossell. Universidad de Almería, España Nina Hidalgo. Universidad Autónoma de Madrid, España Noemí Rodríguez Fernández. Universidad de Oviedo, España Oihane Fernández-Lasarte. Universidad del País Vasco, España Olaia Fontal Merillas. Universidad de Valladolid, España Olga María Alegre de la Rosa. Universidad de La Laguna, España Onowa Reina. Universidad de Sevilla, España Pablo Aparicio Ruiz. Universidad de Sevilla, España Pablo Sánchez Antolín. Universidad de Castilla la Mancha, España Paloma Contreras Pulido. Universidad de Huelva, España Paloma Valdivia. Universidad Autónoma de Barcelona, España Paola Andrea Gómez Montoya. Universidad de Cantabria, España Patricia De Casas. Universidad de Huelva, España Patricia Núñez Gómez. Universidad del País Vasco, España Paula Renes Arellano. Universidad de Cantabria, España Pedro Silva. Escola Superior de Educaçao e Ciencias Sociais-Instituto Politécnico de Leiría, Portugal Próspero Manuel Morán. Universidad de Oviedo, España Rafael Grosso de la Vega. Universidad de Sevilla, España Rafael Rosillo Camblor. Universidad de Oviedo, España Rafael Santana Hernández. Universidad de Las Palmas de Gran Canarias, España Ramón Fernández Pulido. Universidad de Salamanca, España Ramón González Cabanach. Universidad de A Coruña, España Ramón Pérez Pérez. Universidad de Oviedo, España Ramón Tirado. Universidad de Huelva, España Raúl González Fernández. Universidad Nacional de Educación a Distancia, UNED, España Rita Gradaille Pernas. Universidad de Santiago de Chile, Chile Rocío García Carrión. Universidad de Deusto, España Rosa García-Ruiz. Universidad de Cantabria, España Rosa Marchena-Gómez. Universidad de las Palmas de Gran Canaria, España Rosa Maria da Exaltaçao Coutrim. Escola Superior de Educaçao e Ciencias Sociais-Instituto Politécnico de Leiría, Portugal.

Rosario Ortega Ruiz. Universidad Complutense, España Santiago Pozo Sánchez. Universidad de Granada, España Sebastián Guerra San Martín. Pontificia Universidad Católica de Valparaíso, Chile Silvia López Larrosa. Universidad de la Coruña, España Silvia María Redon Pantoja. Pontifica Universidad Católica de Valparaíso, Chile Sonia Peña García. Pontificia Universidad Católica de Ecuador, Ecuador Susana Molina Martín. Universidad de Oviedo, España Susana Rodríguez Martínez. Universidad de A Coruña, España Susana Torío López. Universidad de Oviedo, España Suyapa Martinez Scott. Universidad de Valladolid, España Verónica Marina Guillén Martín. Universidad de Zaragoza, España Vicent Gonzálvez. Universidad de Valencia, España Vicente Manzano-Manzano Arondo. Universidad de Sevilla, España 
Consejo Internacional de Revisores Científicos

Xicu Xabiel García Pañeda. Universidad de Oviedo, España Xose Antón González Riaño. Universidad de Oviedo, España

Yolanda del Cura González. Universidad de Oviedo, España 
AULA ABIERTA • Volumen 49, número 2, abril-junio, 2020

\section{SUMARIO / CONTENTS}

La autopercepción de competencia mediática y su relación con las variables sociodemográficas del profesorado de educación básica

The self-perception of media competence and its relationship with the sociodemographic variables of the teaching staff of three educational institutions located in Lima

Milagros del Carmen Gonzales Miñán, Osbaldo Turpo Gebera y Cristóbal Suárez Guerrero.

La transmisión de valores sociales a través de Internet

The transmission of social values through the Internet abstract

Paula Renés-Arellano, Cleofé Genoveva Alvites-Huamaní y Mari-Carmen Caldeiro-Pedreira.

Prácticas educativas familiares y mediación parental vs dispositivos móviles

Family educational practices and parental mediation vs mobile and internet devices

Antonia Ramírez García y Rocío Gómez Moreno

Aportaciones de expertos del ámbito académico y diseñadores para la creación de experiencias de juego cívicas Contributions from Academic Experts and Game Designers to Develop Civic Game experiences

Jorge Oceja y Natalia González-Fernández.

Validación de una Escala para la Evaluación de las Actitudes hacia la Educación Inclusiva en la formación inicial del educador social

Validation of a Scale for the Assessment of Attitudes towards Inclusive Education in the initial formation of the social educator $M^{a}$ Del Carmen Pegalajar Palomino

¿Es posible la mejora educativa desde los centros? El papel de la organización escolar en los procesos internos de cambio Is it possible to improve education from the schools? The role of the school organisation in the internal processes of change

Noelia Ceballos López, Ángela Saiz Linares.....

Lengua de Signos Española y ámbitos específicos. Una propuesta multidisciplinar e inclusiva para la búsqueda, análisis y creación de Signos

Spanish Sign Language and specific areas. A multidisciplinary and inclusive proposal for the search, analysis and creation of Signs

Aránzazu Valdés-González, Javier Martín-Antón..

La contribución del/de la auxiliar técnico educativo (ATE) para el desarrollo de una plena inclusión

The contribution of the education technical assistant (ATE) for the development of full inclusion

Sebastià Verger Gelabert, Laura Chover, Maria Rosa Rosselló Ramón......

Associated factors with resilience and burnout: a cross-sectional study in a teaching group in Spain

Factores asociados con la resiliencia y el burnout: un estudio transversal en un grupo de docentes en España

$M^{a}$. Inmaculada Vicente De Vera García, $M^{a}$ Inés Gabari Gambarte 


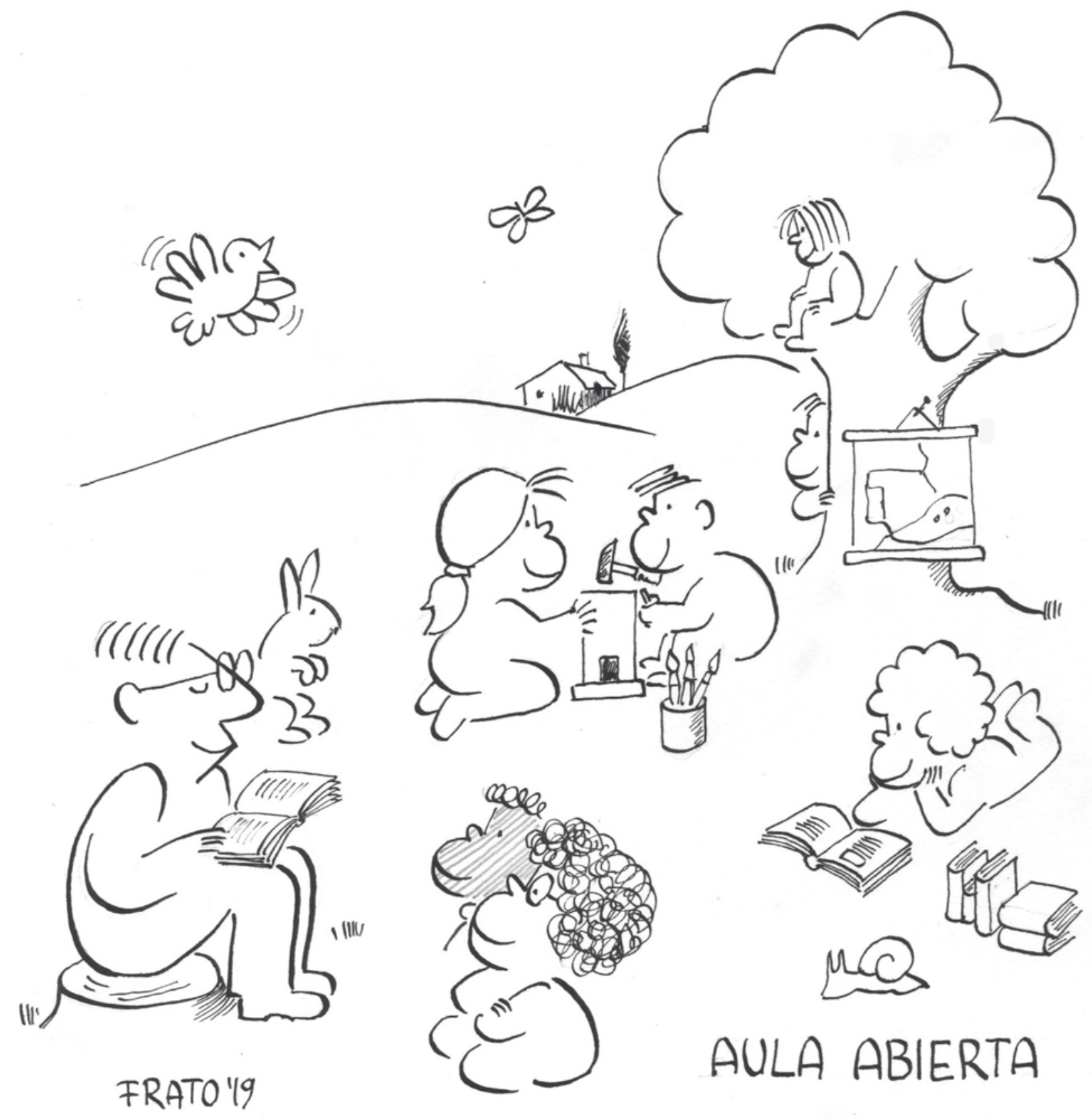


Número 1:

Relación entre Centros Educativos, Familias y Entidades Comunitarias

Schools, Families and Community Institutions Partnership

Coordinado por Dra. Beatriz Rodríguez-Ruiz $\left(^{*}\right)$, Dra. Lucía Álvarez-Blanco $\left(^{*}\right)$, Dra. Raquel-Amaya Martínez-González $\left({ }^{*}\right)$ y Dra. Joyce L. Epstein $\left({ }^{* *}\right)$

${ }^{*}$ ) Universidad de Oviedo - España $\left.{ }^{* *}\right)$ Johns Hopkins University - U.S.

Call for Papers para el número: 31 de agosto de 2018.

Fecha inicial de llamada: 1 de febrero de 2018.

Fecha final de llamada: 31 de agosto de 2018.

Publicación: enero de 2019.

Número 2:

Educación Inclusiva y Tecnologías de la Información y la Comunicación

Inclusive Education and Information and Communication Technologies

Coordinado por José M. ${ }^{a}$ Fernández Batanero (Universidad de Sevilla- España) y Pedro Tadeu (UDI-Instituto politécnico de Guarda - Portugal)

Call for Papers para el número: 1 de enero de 2019.

Fecha inicial de llamada: 1 de noviembre de 2018.

Fecha final de llamada: 1 de enero de 2019.

Publicación: abril de 2019.

Número 3:

Investigación en Educación: avances y retos

Research in Education: progress and challenges

Coordinado por Günter L. Huber, Profesor emérito de la Universidad de Tübingen, Waden - Witenberg, Alemania

Call for Papers para el número: 1 de marzo de 2019.

Fecha inicial de llamada: 1 de noviembre de 2018.

Fecha final de llamada: 1 marzo de 2019.

Publicación: julio de 2019.

Número 4:

Sociedad, Educación e Innovación acelerada: Pertinencia de los Enfoques Sistémicos y Transdisciplinares (Número especial en español e inglés todos los artículos) Society, Education and accelerating Innovation: The Relevance of Systemic and Transdisciplinary Approach (Special number in Spanish and English all articles) Coordinado por Xavier Arreguit (Innobridge, Laussanne, Suiza), Emilio Álvarez-Arregui (Universidad de Oviedo)

Call for Papers para el número: 31 de agosto de 2019.

Fecha inicial de llamada: 1 de noviembre de 2018.

Fecha final de llamada: 31 de agosto de 2019.

Publicación: octubre-diciembre de 2019. 


\section{Publicaciones año 2020:}

Número 1:

Educación Patrimonial

Heritage Education

Coordinado por D. Alex Ibáñez Etxeberría (Universidad del País Vasco - España) y Dña. Olaia Fontal Merillas (Universidad de Valladolid - España).

Call for Papers para el número: 1 de diciembre de 2019.

Fecha inicial de llamada: 1 de noviembre de 2018.

Fecha final de llamada: 1 de diciembre de 2019.

Publicación: enero de 2020

Número 2:

Competencia Mediática. Alfabetización para las nuevas formas de contar con el universo transmedia.i

Coordinado por Dña. Amor Pérez Rodríguez (Universidagd de Huelva - España) y D. Ángel Hernando-Gómez (Universidad de Huelva - España).

Call for Papers para el número: 1 de septiembre de 2019.

Fecha inicial de llamada: 1 de septiembre de 2019.

Fecha final de llamada: 1 de enero de 2020

Publicación: abril de 2020

Número 3:

Aprender a aprender: una competencia clave para la ciudadanía del siglo XXI. Conceptualización, retos, estrategias y evaluación en contextos múltiples.

(Número especial en español e inglés todos los artículos)

Learning to learn: a key competence for 21st century citizenship. Conceptualization, challenges, strategies and evaluation in multiple contexts.

(Special number in Spanish and English all articles)

Coordinado por: Cristina Stringher investigadora de INVALSI (National Italian Institute for Educational Evaluation of Instruction and Training) (Roma - Italia), Ana

Castro Zubizarreta profesora de la Universidad de Cantabria - España) y Maria del Carmen Huerta Guerra investigadora de INVALSI (Roma - Italia).

Call for Papers para el número: 1 de febrero de 2020

Fecha inicial de llamada: 1 de febrero de 2020

Fecha final de llamada: 1 de abril de 2020

Publicación: julio-septiembre de 2020

Número 4:

Avances en Investigación Educativa desde la Teoría y la Práctica

Advances in Educational Research from Theory and Practice

Coordinado por: Günter L. Huber, Universidad de Tübingen, Waden - Witenberg (Alemania), José María Batanero, Universidad de Sevilla (España) y Dolors Forteza

Forteza, Universidad de las Islas Baleares (España).

Call for Papers para el número: 1 de junio de 2020

Fecha inicial de llamada: 1 de junio de 2020

Fecha final de llamada: 15 de septiembre de 2020

Publicación: octubre-diciembre de 2020 